\title{
Cytoprotective Effect and Clinical Outcome of Perioperative Progesterone in Brain Tumors. A Novel Randomized Mono-Centeric Microscopic Evidence Based Research.
}

Mina Maher Raouf ( $\Delta$ drmina2015@gmail.com )

Minia University Faculty of Medicine https://orcid.org/0000-0002-9805-6605

George Abdel-shaheed Hanna

Minia University Faculty of Medicine

Marian Fathi Gayd

Minia University Faculty of Medicine

\section{Research}

Keywords: Cytoprotective effect, clinical outcome, perioperative progesterone, brain tumors, novel randomized mono-centeric, microscopic evidence based research, nuclear neuro-quiescence

Posted Date: September 3rd, 2021

DOl: https://doi.org/10.21203/rs.3.rs-839955/v1

License: (c) (1) This work is licensed under a Creative Commons Attribution 4.0 International License.

Read Full License 


\section{Abstract}

\section{Objectives.}

The primary end-point of the current study was to to provide contemporary estimate about both cytoplasmic and nuclear effects of intramuscular progesterone therapy prior to craniotomy. Secondary end-points were to track post-operative course and short term (3 months) neurological sequele.

\section{Measurments.}

Cellular neuronal microscopic examination by immuno-histochemistry (Progesterone receptor density ) and by H\&E. Allred score for nuclear staining for PR receptors . Post-operative course included time to wean from mechanical ventilation (hours), Length of ICU stay (days) and brain neuro-imaging by brain CT). Short term outcome included sensory, motor and autonomic assessment.

\section{Patients.}

Two hundred fifty two (252) adult patients of both sexes aged 18-60 years, American society of anesthesia (ASA class I-II ) candidate for intra-parenchymal intra-axial elective brain tumors resection , Excluded candidates included refusal to sign document for intervention, urgent craniotomy, history of endometrial or ovarian neoplasia, redo craniotomy, stented coronary arteries or history of deep venous thrombosis.

\section{Design.}

This research was a randomized, double-blind, placebo controlled single center study.

\section{Intervention.}

Two groups, the control group and Progesterone (PR) group. PR group received $1 \mathrm{mg} / \mathrm{kg}$ intramuscular progesterone five days before and five days after craniotomy, while Control group received intramuscular isotonic saline daily, five days before and five days after surgery.

\section{Setting.}

Fixtures performed in Minia university hospital, Neurosurgery operative theatre, Floor two (non trauma floor). The study was registered in local ethical committee on April 2020. First patient enrollment was on first of June 2020 and the patient allocation in the rank ended on 30 of December 2020.

\section{Main results}

Progesterone therapy successfully increased expression of active nuclear PR receptors on oligodendrocytes and astrocytes, provided cytoplasmic and nuclear neuro-quiescence. Progesterone gave better neurological outcome on 3 month follow- up. 


\section{Conclusions.}

Neuronal biopsy examination represented a leap in confirming anti-neuro-inflammatory action offered by progesterone.

\section{Name of the registry. Clinical trial gov.}

Trial registration number. NCT04414020.

Date of registration. $\underline{30}$ of May 2020.

URL of trial registry record. ttps://register.clinicaltrials.gov/prs/app/action/SelectProtocol?

sid=S0009WLW\&selectaction=Edit\&uid=U0004XC5\&ts=2\&CX=-vrqng $\underline{9}$

\section{Introduction}

Progesterone is a naturally occuring neurosteroid that can offer neuroprotective effects via pleiotropic pathways. Progesterone decreases cerebral oedema (1) restores blood-brain barrier integrity (2), reduces the inflammatory responseand prevents cellular necrosis and apoptosis. The compression of brain tissue by a tumour mass is believed to be a major cause of the clinical symptoms seen in patients with brain cancer. However, the biological consequences of these physical stresses on brain tissue are unknown. Here, via imaging studies in patients and by using mouse models of human brain tumours, we show that a subgroup of primary and metastatic brain tumours, classified as nodular on the basis of their growth pattern, exert solid stress on the surrounding brain tissue, causing a decrease in local vascular perfusion as well as neuronal death and impaired function. We demonstrate a causal link between solid stress and neurological dysfunction by applying and removing cerebral compression, which respectively mimic the mechanics of tumour growth and of surgical resection. We also show that, in mice, treatment with lithium reduces solid-stress-induced neuronal death and improves motor coordination. Our findings indicate that brain-tumour-generated solid stress impairs neurological function in patients, and that lithium as a therapeutic intervention could counter these effects.

Brain tissue can be deformed and its function perturbed by two mechanical forces: tumour-associated edema and solid stress. Solid stress refers to the compressive and tensile mechanical forces exerted by the solid components of the tissues, such as cells and extra-cellular matrix The tumour growth-induced deformation of the brain - the so-called "mass effect" .(3)

Primary outcome was to examine cytoplasmic and nuclear actions of intra-musculer progesterone amid surgical neuronal injury and tumor induced neuro-excitotoxicity. Secondery outcome was to explore short term outcome 3 months after craniotomy regarding sensory, motor and autonomic function.

\section{Material And Methods}


This prospective randomized double-blind mono- center study approved by the local ethics committee of the Faculty of Medicine, Minia University hospital under institutional review board number ( $\underline{\mathbf{6 2 3}}-\mathbf{4} / \mathbf{2 0 2 0})$, and registered on clinical trial (NCT04414020), involved 252 adult patients of both sexes, ASA I-II, who underwent elective craniotomy. Exclusion criteria included candidate refusal to participate in the study, emergency craniotomy, any risk for developing venous thromb-embolism (previous deep venous thrombosis, systemic lupus or cerebrovasculer stroke), tumor recurrence, population with stented coronary arteries, morbid obesity and giving relevant history of ovarian or endometrial neoplasia.

An informed written consent obtained from all participants who were randomly and equally allocated into two groups through web-based randomizer (https://www.randomizer.org). Computerized randomization was performed using variable block sizes. Opaque sealed envelopes were used for allocation concealment. Boxes with patient numbers containing unlabeled coded vials were provided for all patients. Saline prepared for injection was in equi-volume with progesterone. Serum progesterone was checked twice, first immediately before initiating first dose and the second one was taken at one week after therapy. Double blind fashion disputed (Patient allocation was concealed from patient and the histopathologist). Data acquisition was tasked by two anesthetic lecturers who were not informed about study design. Candidates were assigned in two equal groups, Control group received intramuscular saline $(2 \mathrm{ml}-\mathrm{NaCL} 0.9 \%)$ as a placebo for five days daily before and after surgery, while progesterone group (PR group) received intramuscular progesterone $(1 \mathrm{mg} / \mathrm{kg})$ for five days daily before and after craniotomy. Saline prepared for injection was in equi-volume with progesterone.

Both injectate, progesterone and placebo are similar regarding color, duration of therapy and route of injection. Serum progesterone was checked twice, first immediately before initiating first dose and the second one was taken at surgery morning. In both groups, general anesthesia and endotracheal intubation was accomplished with Propofol $(2 \mathrm{mg} / \mathrm{kg})$ and Fentanyl $(2 \mathrm{mcg} / \mathrm{kg})$ and neuromuscular blockade by cis-atracurium $(0.5 \mathrm{mg} / \mathrm{kg})$. Anesthesia was maintained with sevoflurane (2-4 \%). Intraoperative monitoring included 5 lead elecrtocardiogram, invasive blood pressure, temperature probe, oxygen saturation, exhaled $\mathrm{CO}_{2}$ (end-tidal capnography), train of four and bispectral index. Tube of polyvinyl chloride size 7 secured at the angle of the mouth with a stabilizer. Fixation decided to be on mouth angle opposite to surgical site. Full sensory and motor neurological examination were executed on 3 months visit post-operative.

\subsection{Surgical biopsy.}

Tumors were excised following standard surgical techniques regarding positioning, skin incision, craniotomy, dural opening, dissection, hemostasis and closure. Biopsy obtained from multiple points at the tumor-brain interface by cotton tipped swab under cover of surgical microscope.

\subsection{Histopathology examination}

\subsubsection{Case selection and tissue sample preparation}


Paraffin blocks with clinico-pathological data of the patients were collected. H\&E slides were prepared to detect histopathological changes as congestion, cytoplsmic edema, necrosis and inflammation.

\subsubsection{Immunohistochemistry.}

Serial sections were cut $4 \mu \mathrm{m}$ thick on positively charged slides. The slides were de-paraffinized with xylene, rehydrated through graded ethyl alcohol. Then, they were immersed in $3 \%$ hydrogen peroxide for $30 \mathrm{~min}$ and rinsed in phosphate buffer solution (PBS). Citrate buffer $(\mathrm{pH} 6.0)$ was used for antigen retrieval by the microwave for 10 minutes. Sections were left to cool at room temperature then washed in PBS. Afterwards, rabbit polyclonal anti-PR anti-body (ready to use, Abcam) was incubated overnight at $4^{\circ} \mathrm{C}$ in humidity chamber. Slides were then rinsed with PBS before applying secondary antibody for 30 min. After wash in PBS, streptavidin-biotin complex was added for another30 min. Brownish color developed by using diamino-benzoate (DAB), then the slides were washed in distilled water. Lastly, they were stained with hematoxylin, dehydrated, cleared by xylene and covered slipped.

\subsubsection{Evaluation of immunostaining.}

According to Allred scoring system, The intensity score (IS) of nuclear staining evaluated as follows: $0=$ no positive cells, $1=$ mild positivity, $2=$ moderate positivity and $3=$ strong positivity. The proportion of staining power evaluated according to the percentage of positive cells: $0=$ negative, $1=1 \%, 2=2-35 \%$, $3=36-65 \%$ and $4=66-100 \%$. PR immune-expression score was calculated as the algebric summation of the scores for the intensity of staining and the extent of staining power. Final scores were then classified into low $(<3)$ and high $(\geq 3)(0)$.

\subsection{Statistical analysis}

\subsubsection{Sample Size Calculation:}

Before the study runover, the number of patients required in each group was determined after a power calculation according to data obtained by a Pilot study performed on ten consented candidates, five in each group. In that study, the percentage of cellular damage in Progesterone group was $20 \%$ (one case with cytoplasmic oedem), while in Placebo control group was $80 \%$ (four cases with cytoplasmic degeneration). A sample size of $\underline{\mathbf{1 2 6}}$ patients in each group was determined to provide $99 \%$ power for Fisher's exact test at the level of 0.05 significance using G Power 3.19.2 software.

\section{Figure 1 reflecting sample size calculation at power $99 \%$.}

Exact - Proportions: Inequality, two independent groups (Fisher's exact test)

Options: Exact distribution

Analysis: A priori: Compute required sample size

Input: Tail(s) = Two




\begin{tabular}{|c|c|c|}
\hline & Proportion p1 & $=0.2$ \\
\hline & Proportion p2 & $=0.8$ \\
\hline & a err prob & $=0.05$ \\
\hline & Power (1- $\beta$ err prob) & $=0.99$ \\
\hline & Allocation ratio N2/N1 & $=1$ \\
\hline Output: & Sample size group 1 & $=126$ \\
\hline & Sample size group 2 & $=126$ \\
\hline & Total sample size & $=252$. \\
\hline
\end{tabular}

\section{Statistical analysis}

- The collected data were coded, tabulated, and statistically analyzed using SPSS program (Statistical Package for Social Sciences) software version 20.

-Descriptive statistics were done for Parametric quantitative data by mean, standard deviation and minimum \& maximum of the range, while they were done for categorical data by number and percentage.

-Analyses within each group were done for parametric quantitative data using paired sample $t$ test, and for qualitative data using Wilcoxon signed rank test. Analyses were done for qualitative data using Fisher Exact test.

-The level of significance was taken at $(P$ value $<0.05)$.

\section{Results}

Table (1) shows the brain CT(1) on admission before starting therapy, CT (2) immediately preoperative, CT (3) 72 hours post-craniotomy, extubation time after craniotomy, Biopsy by $\mathrm{H}$ and $\mathrm{E}$ and 3 months postoperative follow up. Basal pre-operative brain CT data revealed statistical insignificant data comparing two groups ( $P$ value $\mathbf{0 . 2 4 1}$ ). Statistically significant difference in-between two groups noticed in CT2, CT3, extubation time and biopsy examination. In PR group, Brain imaging in CT2 exploits 112 case $(88.8 \%)$ showing free scanning and 14 case $(11.2 \%)$ with mild peri-lesional brain oedema, On the other hand, Control group presents with 18 case (14.2\%) with mild peri-lesional brain oedema, 14 case (11.2\%) with moderate diffuse brain oedema and 94 cases (74.6\%) with free scanning, CT3 in PR group exploits number of free brain CT cases increase to 117 case (92.8\%). Significant statistical difference between basal CT ,CT2 and CT3 inside PR group ( $P$ values $=0.003$ and 0.004 respectively), Eosinophilic infiltration was comparable inbetween two groups (Pvalue= 0.9 ) Slides with free examination featured in PR group (116-while no statistical significant difference between basal values, CT2 and CT3 values inside 
Control group ( $\mathbf{P}$ values $\mathbf{= 0 . 0 7}$ and $\mathbf{0 . 0 8}$ ). Three cases $(2.5 \%$ ) only declared to have mild oedema around tumor margin and $6(5 \%)$ cases with moderate brain oedema. Control group brain imaging revealed 11 case (8.7\%) with mild peri-lesional brain oedema, 19 cases (15.2\%) with moderate diffuse brain oedema, normal brain CT imaging declared in 96 cases $(76.1 \%)$.

Microscopic examination revealed statistical significant difference between two groups . Perivascular necrosis, cytoplasmic oedema, neutrophilic infiltration and hydrophilic degeneration were more featured in Control group rather than PR group ( $P$ values $=0.003,0.004,0.03,0.05$ respectively $)$. Slides with free examination were featured in PR group (116 candidates-92.1\%) much more than Control group ( 72 cadidates- $57.1 \%)$.

It took much less time to wean 119 case (92.3\%) from mechanical ventilation post-craniotomy in PR group (less than 3 hours). Only seven cases rested on mechanical ventilation for 5 hours postoperative then weaned safely. Twenty five (19.9\%) candidate in Control group fullfiled criteria for extubation after craniotomy in more than 3 hours ( from five to seven hours), 101 population (80.1\%) weaned within 3 hours.

Biopsy examination under $\mathrm{H}$ and $\mathrm{E}$ stain was free of signs of injury in 116 case $(92.1 \%)$ case in PR group, ten cases (7.9\%) only gave microscopic features of cytoplasmic hydrophilic degeneration, Control group was rich in cytoplasmic injury signs as peri-vasculer hemorrhage (15 case, $11.9 \%$ ), severe cytoplsmic oedema (9 cases,7.1\%), esinophilic infiltration (5 cases, 3.9\%), dense neutrophilic infiltration (12 case, $9.5 \%$ ) and 13 case (10.3\%) with hydrophilic degeneration. Three months follow up was fair enough in favour of PR group with statistical significant difference ( $P$ value $=\mathbf{0 . 0 0 1}$ ) presenting 121 case (96\%) with free neurological outcome, 5 candidates with either loss light reflex, agraphia or fluent aphasia. Control group was obviously showed cases with neurological insults ( 10 cases with cerebeller ataxia , 5 cases with loss of light reflex, 6 case with agraphia and 2 case with fluent aphasia.

Table (2) presents compartive data regarding age, intraopertive blood loss, serum progesterone before and after therapy and length of ICU stay. Significant difference was clear in ICU stay ( $P$ value $=0.0001$ ) being shortened in PR group (28.6 \pm 9.6 hours) however it was quite doubled in control group (56.3 \pm 16.5hours). Data regarding age, introperative blood loss and serum progesterone were comparable.

Table (3) presenting intensity and proportion of progesterone receptors in both groups. There is a highly statistical significant difference in between 2 groups being higher in PR group ( score $>3$ ).

\section{Disscussion}

Neuro-critical care residents questioned for a safe effective management for trauma or surgical induced neuronal injury. Biopsy based microscopic and immuno-histochemistry examination is a leap unique method that doesnot give chance for bias. Extensive literatures had led to a debate about the clinical efficacy of progesterone in providing neuro-quiescence and neuronal repair depending only on clinical 
course and neurological outcome (4). Novelty of this paper came from its use of neuronal biopsy, a new senior modality to deny or confirm cellular injury.

Despite the multi-factorial benefits of progesterone obtained in the experimental models of TBI and the promising results of two Phase II clinical trials $[5,6]$, two Phase III clinical trials failed to show benefits of progesterone $[7,8]$. Among the concerns that have been raised were the diversity of the enrolled patients concerning sex, age, and severity of TBI and the different doses regimen of progesterone used.

Many recent nested randomized clinical trials reported that progesterone can provide cerebroprotection. Zhao etal 2019 (9), in a wide spectrum meta-analysis consisting of eight randomized clinical trial hosted 2251 patients were fixtured for both safety and efficacy of injected progesterone on TIBS population.

The regimen included $1 \mathrm{mg} / \mathrm{kg}$ intramuscular progesterone every 12 hours for five days. Their results are compliant with us regarding candidates with progesterone group had less ICU time, better neurologic outcomes $(\mathrm{RR}=1.51 ;, P=0.007)$ than those who received placebo. Progesterone offered neuroprotection till 3 months after impact.

Neuronal injury in operable brain tumors is coming from two ways, first is the direct mechanical compression by the tumor itself impeding adjacent healthy tissue blood supply and the other is surgical craniotomy. Surgical craniotomy induced cerebral injury is mostly due to direct parenchymal injury resulting from focal exertion of pressure on retracted neural tissue associated with the use of plate and self-sustaining retractors to access deep intra-parenchymal lesions. New methods were in use to minimize tissue injury including neuro-navigation and endoscopic approaches, but yet no full protection was declared. This was the point of interest dealt by Stephen etal., 2020(10) who linked post-craniotomy morbidity and mortality related to retractors induced ischemic injury. Patho-physiological mechanisms that can provoke neuronal injury following surgical head trauma is post-traumatic inflammation, which has been shown to increase blood- brain barrier (BBB) permeability and cerebral edema. During posttraumatic inflammation, metabolic products of arachidonic acid, known as prostanoids, including prostaglandins, prostacyclin and thromboxanes, are released. Following the overwhelming production of inflammatory mediators within the injured brain is believed to contribute to the cerebral damage and BBB dysfunction (11). Key mechanism in ameliorating surgery induced cerebral injury is combined action of progesterone and its active metabolite allo-pregnanolone

Results of preclinical analyses demonstrated that allo-pregnanolone is a potent inducer of neural progenitor proliferation of progenitor cells in vitro.

Liere etal., 2014 (12) in their paper about revisting the role of progesterone metabolite in central nervous system. Allopregnanlone significantly increased neurogenesis within the subgranular zone of the dentate gyrus and subventricular zone, maintaining selective controlled BBB permeability, promoted survival of young neurons and functional recovery(12). This was clearly identified in PR group biopsy slides and brain CT. Biopsy showed impedence to neutrophilic infiltration and blood vessel congestion. Also brain 
CT was demonstrative in PR group regarding decrease in perilesional oedema( cytotoxic brain oedema surrounding tumor). This action is explained by progesterone inhibitory action on inflammatory vasodilator substances (Nitric oxide and Interleukins) and by increasing endothelial progenitor cells (13)

Dose choice was based upon a previous randomized controlled trials that used progesterone in traumtic brain injury in a dose $1 \mathrm{mg} / \mathrm{kg}$ intramusculer for 5 days post-traumatic targeting length of ICU stay as a primary endpoint. (Hassan etal., 2017).

The current research endorsed enhanced transcription and upregulation of nuclear Progesterone receptors with enhanced uptake of staining ( both intensity and proportion are increase) in PR group. Candidates with these findings showed smooth postoperative course (less time for weaning and ICU stay). Both progesterone can suppress inflammatory mediators productions together with the gamma amino butyric acid (GABA) like action of its active metabolite allopreganolone which can bind to GABAa receptors mediating neuronal hyperpolrization via opening of inhibitory chloride channel. This GABA mimetic action increase neuronal level of inhibitory neurotransmitter glycine that counteract excitatory effects of glutamate that increase neuronal injury induced by tumor and craniotomy $(15,16)$.

Our results run hand by hand with Hassan etal.,2017 (17) who injected progesterone intramuscularly in traumatic brain injury victims for five days declaring less time for ICU stay and early weaning from mechanichal ventilation.

\section{Limitations}

1. Excluding emergency craniotomy.

2. Not all cases cooperated in biopsy harvesting .

3. Surgical hazards from biopsy obtaining.

\section{Declarations}

\section{Author Contribution}

1. Mina Maher Raouf . Mauscript drafting, conceptualization and data collection.

2. George Abdel-shaheed. Data analysis.

3. Marian Fathi Gayyd. Histopathological examination.

\section{Acknowledgement.}

1. Mohab M. Naguib.

Lecturer of neurosurgery. Minia university hospital. Minia. Egypt.

Role; Harvesting neuronal biopsy, data collection, neurological follow up. 


\section{Ethics approval and consent to participate.}

Institutional review approval waived on number (623-4-2020).

\section{Availability of data and materials}

\section{ALL data supporting results ( biopsy) in the current research sent with submission for clarity. CT images sent upon request.}

\section{Competing interests}

\section{No competing interest}

\section{Funding}

No specific source of funding

\section{Authors' information}

All authors whose names appear on the submission

1) Made substantial contributions to the conception or design of the work; or the acquisition, analysis, or interpretation of data; or the creation of new software used in the work;

2) Drafted the work or revised it critically for important intellectual content;

3) Agree to be accountable for all aspects of the work in ensuring that questions related to the accuracy or integrity of any part of the work are appropriately investigated and resolved.

\section{References}

1- Wang X, Zhang J, Yang Y, et al. Progesterone attenuates cerebral edema in neonatal rats with hypoxicischemic brain damage by inhibiting the expression of matrix metalloproteinase- 9 and aquaporin-4. Exp Ther Med. 2013;6(1):263-267.

2-. He L, Zhang $X$, Wei $X$ etal... Progesterone attenuates aquaporin-4 expression in an astrocyte model of ischemia/reperfusion. Neurochem Res. 2014;39(11):2251-2261.

3-. Chen G, Shi JX, Qi M et al.,. Effects of progesterone on intestinal inflammatory response, mucosa structure alterations, and apoptosis following traumatic brain injury in male rats. J Surg Res. 2008;147(1):92-98.

4- Stylianopoulos $T$ et al. Causes, consequences, and remedies for growth-induced solid stress in murine and human tumors. Proc Natl Acad Sci U S A 109, 15101-15108, doi:10.1073/pnas.1213353109 (2012). 
5-Wright D., Kellermann A., Hertzberg V., etal, A randomized clinical trial of progesterone for acute traumatic brain injury.Ann Emerg Med. 2007 Apr; 49(4):391-402.

6- Xiao G, Wei J, Yan W etal.,. Improved outcomes from the administration of progesterone for patients with acute severe traumatic brain injury: a randomized controlled trial. Crit Care. 2008; 12(2):R6.

7- Wright D., Kellermann A., Hertzberg V., et al. ProTECT: A randomized clinical trial of progesterone for acute traumatic brain injury. Ann Emerg Med. 2007::391-402.

8- Skolnick BE, Maas A., Narayan R. etal,. A clinical trial of progesterone for severe traumatic brain injury. N Engl J Med. 2014 Dec 25; 371(26):2467-76.

9- Pan Z, Zhao Y, Huang W, etal,. Effect of progesterone administration on the prognosis of patients with severe traumatic brain injury: a meta-analysis of randomized clinical trials.2019.Volume 13.265-273.

10-Stephen Z , Kenneth A., Samuel M., etal,.. Use of Vycor Tubular Retractors in the Management of Deep Brain Lesions: A Review of Current Studies. World Neurosurg 2020 Jan;133:283-290.

11- Si D, Li J, Liu J, et al. Progesterone protects blood-brain barrier function and improves neurological outcome following traumatic brain injury in rats. Exp Ther Med. 2014;8(3):1010-1014.

12- Guennoun R, Labombarda F, Gonzalez D. etal,.. Progesterone and allopregnanolone in the central nervous system: response to injury and implication for neuroprotection.

13. Lei B, Mace B, Dawson HN, etal,.. Anti-inflammatory effects of progesterone in lipopolysaccharidestimulated BV-2 microglia. PLoS One. 2014;9(7):e103969.

14- Fitzgibbons PL, Dillon DA, Alsabeh R, etal,.. Template for reporting results of biomarker testing of specimens from patients with carcinoma of the breast. Arch Pathol Lab Med. 2014 May;138(5):595-601.

15- Djebaili M, Hoffman SW, Stein DG. Allopregnanolone and progesterone decrease cell death and cognitive deficits after a contusion of the rat pre-frontal cortex. Neuroscience. 2004;123:349-359.

16- Wright D., Kellermann A., Hertzberg V., et al. ProTECT: a randomized clinical trial of progesterone for acute traumatic brain injury. Ann Emerg Med. 2007::391-402.

17- Hassan A Aboukhabar, Amr A, etal,.. Impact of progesterone administration on outcome in patients with severe traumatic brain injury.vol4.2017.p84;89.

\section{Tables}

Table (1) Brain computerized tomography, Extubation time, Biopsy by $\mathrm{H}$ and $\mathrm{E}$ and 3 months neuronal follow up (Data expressed as mean \pm standered deviation). 


\begin{tabular}{|c|c|c|c|}
\hline \multirow[t]{2}{*}{ Variable } & \multicolumn{2}{|c|}{ Groups } & \multirow{2}{*}{$\begin{array}{c}\chi^{2} \\
\text { P values }\end{array}$} \\
\hline & $\begin{array}{l}\text { PR group } \\
\quad(126)\end{array}$ & $\begin{array}{c}\text { Controls } \\
\text { (126) }\end{array}$ & \\
\hline CT1 (basal): & $97(71.5 \%)$ & $90(71.4 \%)$ & 0.241 \\
\hline Free & $20(15.8 \%)$ & $19(15.2 \%)$ & \\
\hline Mild & $9(13.3 \%)$ & $17(13.4 \%)$ & \\
\hline \multicolumn{4}{|l|}{ Moderate } \\
\hline CT2: & \multirow{2}{*}{$\begin{array}{r}112 \\
(88.8 \%)\end{array}$} & $94(74.6 \%)$ & \multirow[t]{5}{*}{$0.0001 *$} \\
\hline Free & & $18(14.2 \%)$ & \\
\hline Mild & $\begin{array}{r}14 \\
(11.2 \%)\end{array}$ & $14(11.2 \%)$ & \\
\hline Moderate & \multirow{2}{*}{\multicolumn{2}{|c|}{$0.003 * *$}} & \\
\hline Pvalue** & & & \\
\hline \multirow{5}{*}{$\begin{array}{l}\text { CT3: } \\
\text { Free } \\
\text { Mild } \\
\text { Moderate } \\
\text { Pvalue** }\end{array}$} & \multirow{5}{*}{$\begin{array}{r}117 \\
(92.8 \%) \\
3(2.5 \%) \\
6(4.7 \%) \\
0.004 * *\end{array}$} & $96(76.1 \%)$ & \multirow[t]{5}{*}{$0.001 *$} \\
\hline & & $11(8.7 \%)$ & \\
\hline & & $19(15.2 \%)$ & \\
\hline & & \multirow{2}{*}{0.08} & \\
\hline & & & \\
\hline \multirow{7}{*}{$\begin{array}{l}\text { Biopsy: } \\
\text { Free } \\
\text { Perivascular hge \& early necrosis } \\
\text { Severe cytoplasmic oedema } \\
\text { Eosinophilic infiltration } \\
\text { Dense neutrophil infiltration } \\
\text { Hydrophilic degenration }\end{array}$} & \multirow{2}{*}{$\begin{array}{r}116 \\
(92.1 \%)\end{array}$} & $72(57.1 \%)$ & $0.005^{*}$ \\
\hline & & $15(11.9 \%)$ & $0.003 *$ \\
\hline & 0 & $9(7.1 \%)$ & $0.004 *$ \\
\hline & 0 & $5(3.9 \%)$ & 0.9 \\
\hline & 4 & $12(9.5 \%)$ & $0.003^{*}$ \\
\hline & & $13(10.3 \%)$ & \multirow[t]{2}{*}{$0.05^{*}$} \\
\hline & & & \\
\hline \multirow{5}{*}{$\begin{array}{l}3 \text { months examination: } \\
\text { Free } \\
\text { Cerebellar ataxia } \\
\text { Others (loss of light reflex, agraphia \& fluent } \\
\text { aphasia) }\end{array}$} & \multirow{5}{*}{$\begin{array}{r}121 \\
(96.0 \%) \\
0 \\
5(4 \%)\end{array}$} & $103(81.7 \%)$ & \multirow{5}{*}{$\begin{array}{r}\mathbf{0 . 0 0 1 *} \\
\text { (Fischer's } \\
\text { exact test) } \\
\mathbf{0 . 0 0 4 *} \\
\mathbf{0 . 0 0 3 ^ { * }}\end{array}$} \\
\hline & & $10(7.9 \%)$ & \\
\hline & & \multirow{3}{*}{13 (10.4\%) } & \\
\hline & & & \\
\hline & & & \\
\hline Extubation time: & $\begin{array}{r}119 \\
(92.3 \%)\end{array}$ & $\begin{array}{r}101 \\
(80.1 \%)\end{array}$ & $0.0001 *$ \\
\hline Within 3 hours & $7(7.7 \%)$ & 25 (19.9\%) & \\
\hline More than 3 hours & & & \\
\hline
\end{tabular}

*Wilcoxon signed-rank test for the intragroup data (against . baseline CT ), **MannWhitney U-test (change between groups). ${ }^{* *} \boldsymbol{P}<0.05$ is considered significant for intragroup comparison $* \boldsymbol{P}<0.05$ is considered significant for comparison between groups.

*Significance difference inbetween two studied groups ( $\mathrm{p}$ value $<0.05$ ) 
** Significance difference between basal and other CT readings in studied groups( $\mathrm{p}$ value $<0.05)$

CT1= Brain CT accomplished before therapy initiation ( on admission )

CT2= Brain CT accomplished one week after therapy ( on surgery morning)

CT3= Brain CT accomplished 3 days postoperative.

Extubation time= Time elapsed from transfer from operative theatre to neuro-ICU till weaning from assissted ventilation to spontaneous ventilation.

Table (2) : Demographic and operative data (intraoperative blood loss, serum

progesterone before and after therapy and ICU stay, data expressed as mean \pm standered deviation).

\begin{tabular}{|c|c|c|c|c|}
\hline Variable & PR group (126) & Controls (126) & & $P$ value \\
\hline Age (years) & $50 \pm 12 \quad(38-62)$ & $52 \pm 9(43-61)$ & & 0.517 \\
\hline \multirow[t]{2}{*}{ Sex } & M.(102-80.9\%). & M.(92-73\%). & & 0.312 \\
\hline & F. $(24-19.1 \%)$. & F. $(34-37 \%)$ & & 0.261 \\
\hline $\begin{array}{l}\text { Serum progesterone (before) } \\
\text { (ng/L). }\end{array}$ & $\begin{array}{r}0.19 \pm 0.1(0.09- \\
0.29)\end{array}$ & $0.177 \pm 0.09(\underset{0.08-}{0.26)}$ & & 0.588 \\
\hline $\begin{array}{l}\text { Serum progesterone } \\
\text { (after) }(\mathbf{n g} / \mathbf{L})\end{array}$ & $\begin{array}{r}0.48 \pm 0.15(0.33- \\
0.63)\end{array}$ & $\begin{array}{r}0.52 \pm 0.15(0.37- \\
0.67)\end{array}$ & 0.423 & \\
\hline ICU stay ( hours) & $\begin{array}{r}28.6 \pm 9.6(19- \\
38.2)\end{array}$ & $56.3 \pm 16.5(39.8-$ & & $0.0001 *$ \\
\hline $\begin{array}{l}\text { Intraoperative blood loss } \\
(\mathrm{ml}) \text {. }\end{array}$ & $650 . \pm 200\left(\begin{array}{r}450- \\
850)\end{array}\right.$ & $\begin{array}{r}600 \pm 350(250- \\
900)\end{array}$ & & 0.072 \\
\hline
\end{tabular}

*done with independent sample $t$ test.

*P value significant when less than or equal 0.005

Table (3)_presenting intensity and proportion of progesterone receptors in both groups.

\begin{tabular}{|lccc|}
\hline Variable & PR group(126) & Controls(126) & $\begin{array}{c}\text { t } \\
\text { P value }\end{array}$ \\
\hline Intensity score and staining proportion & $5 \pm 1.4$ & $2.5 \pm 1.5$ & 6.363 \\
& & & $\mathbf{0 . 0 0 0 1 *}$ \\
\hline
\end{tabular}

*done with independent sample $t$ test.

\section{Figures}




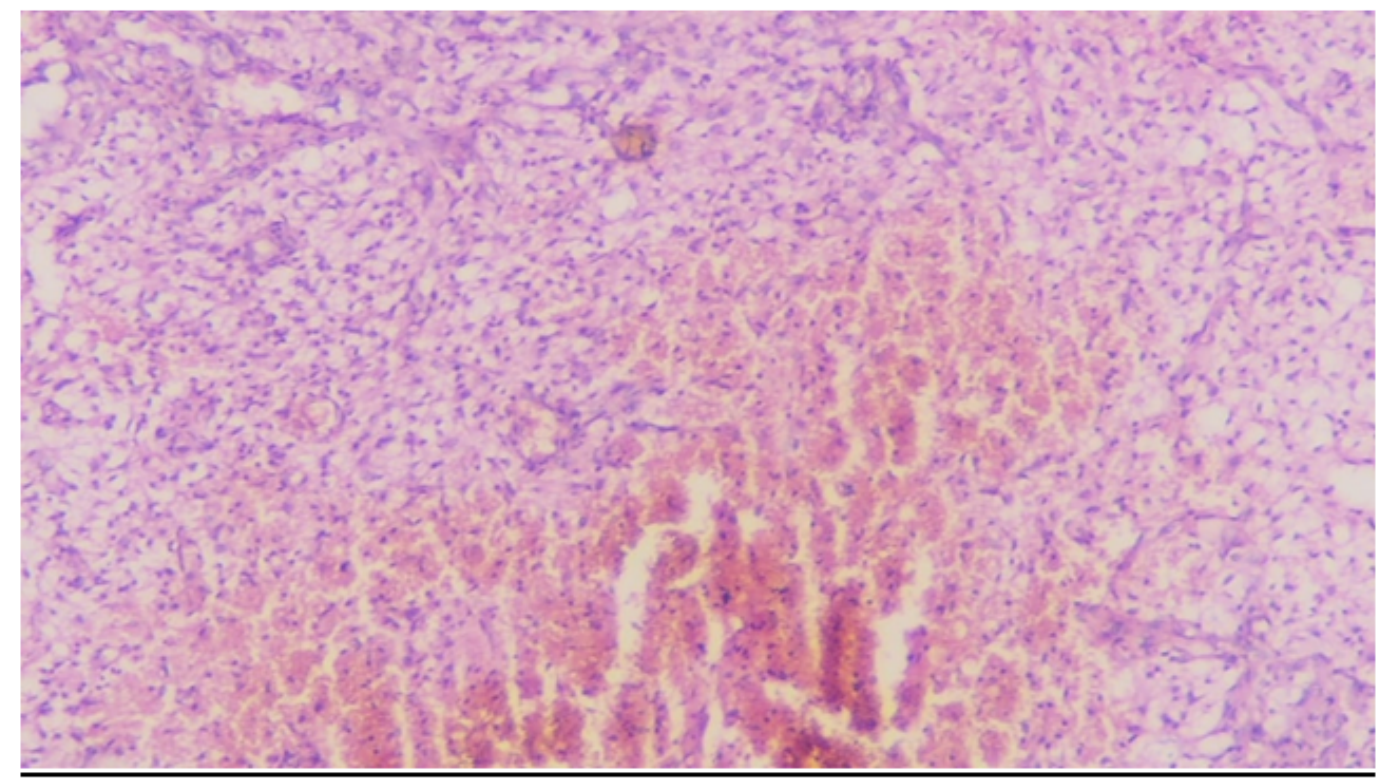

A

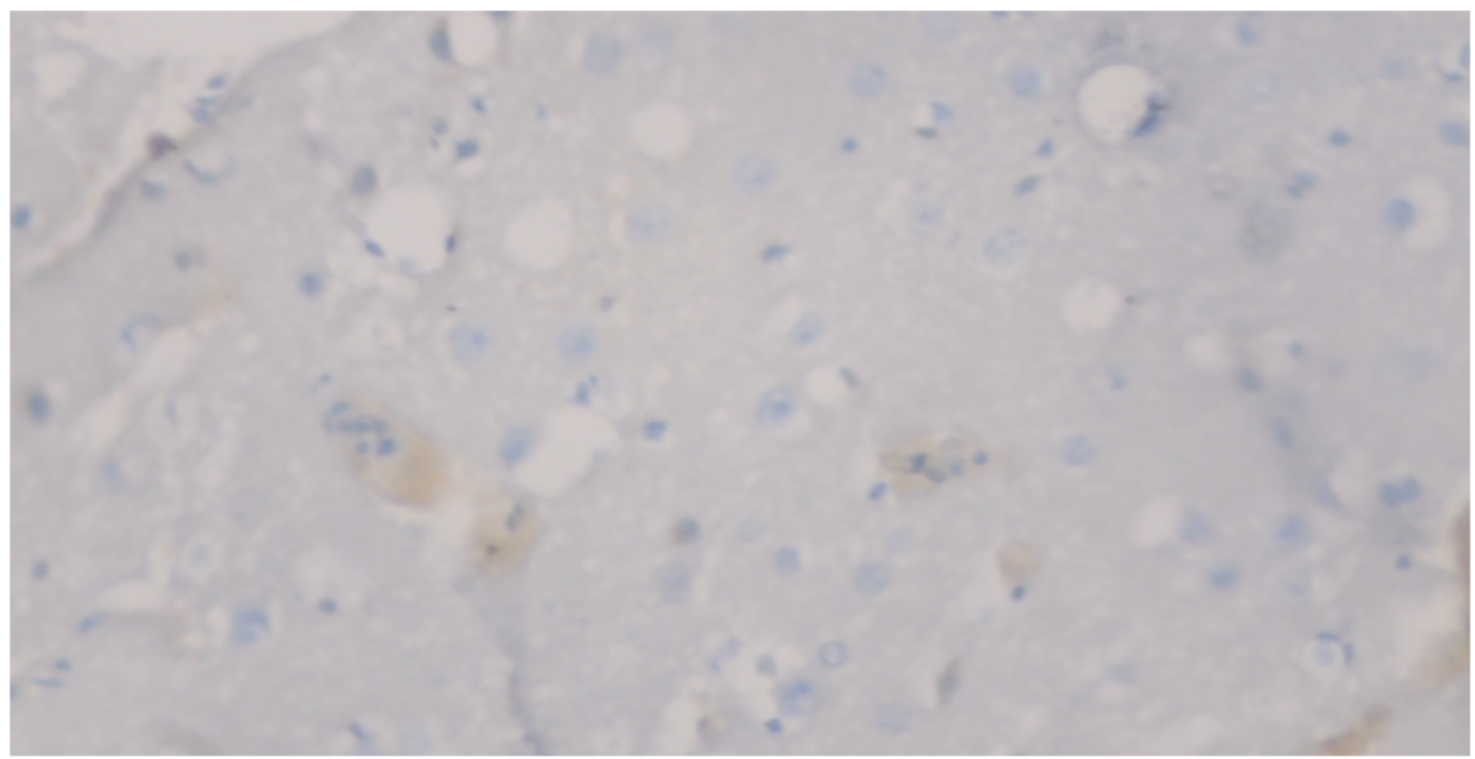

B

\section{Figure 1}

(A) by $\mathrm{H}$ and $\mathrm{E}$ stain of a patient in control group ( 44 years old male patient, temporal glioma). The slide shows ruptured blood vessels, mild hydrophilic degeneration. Brain imaging 3 days post-craniotomy revealed moderate brain oedema, Three month follow up was free. (B) of the same patient by immunohistochemistry, no PR receptors . 


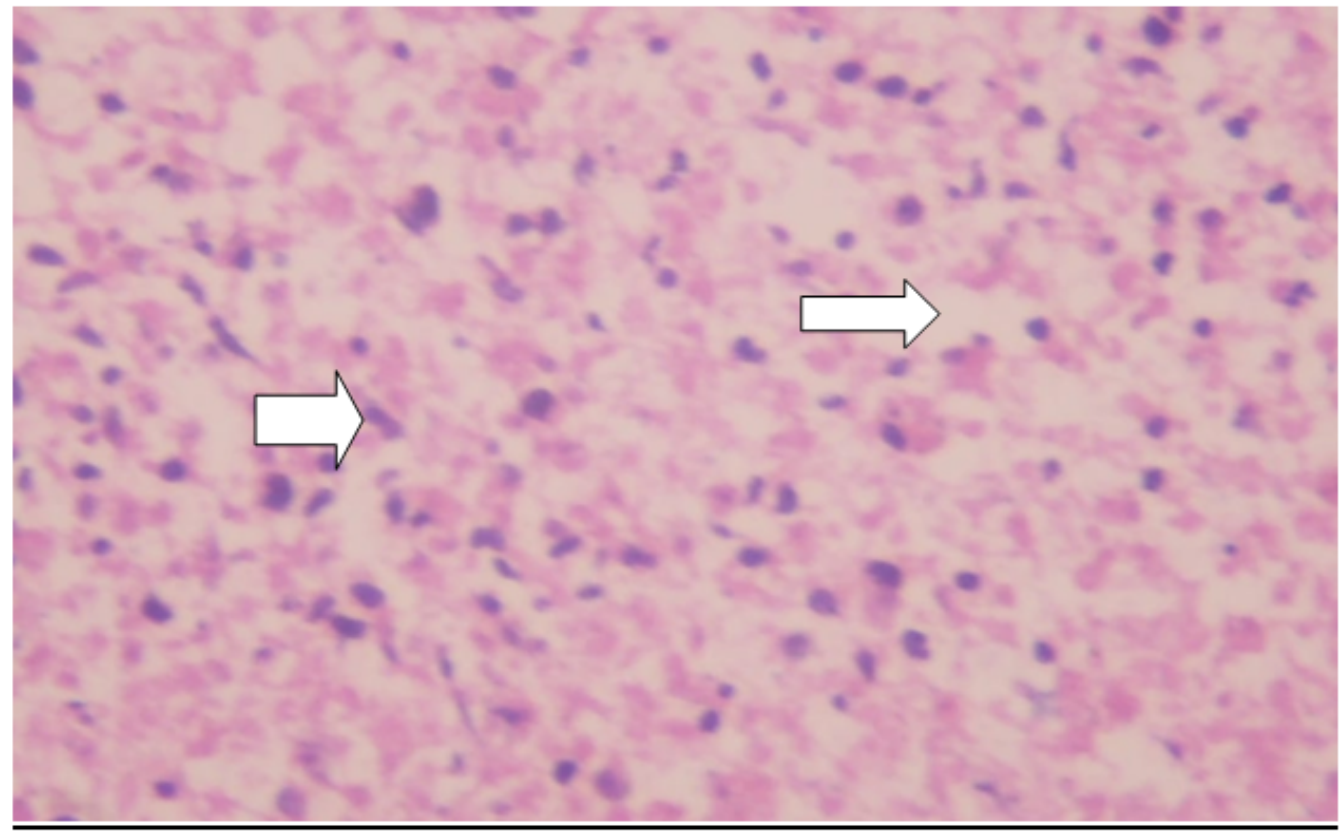

A

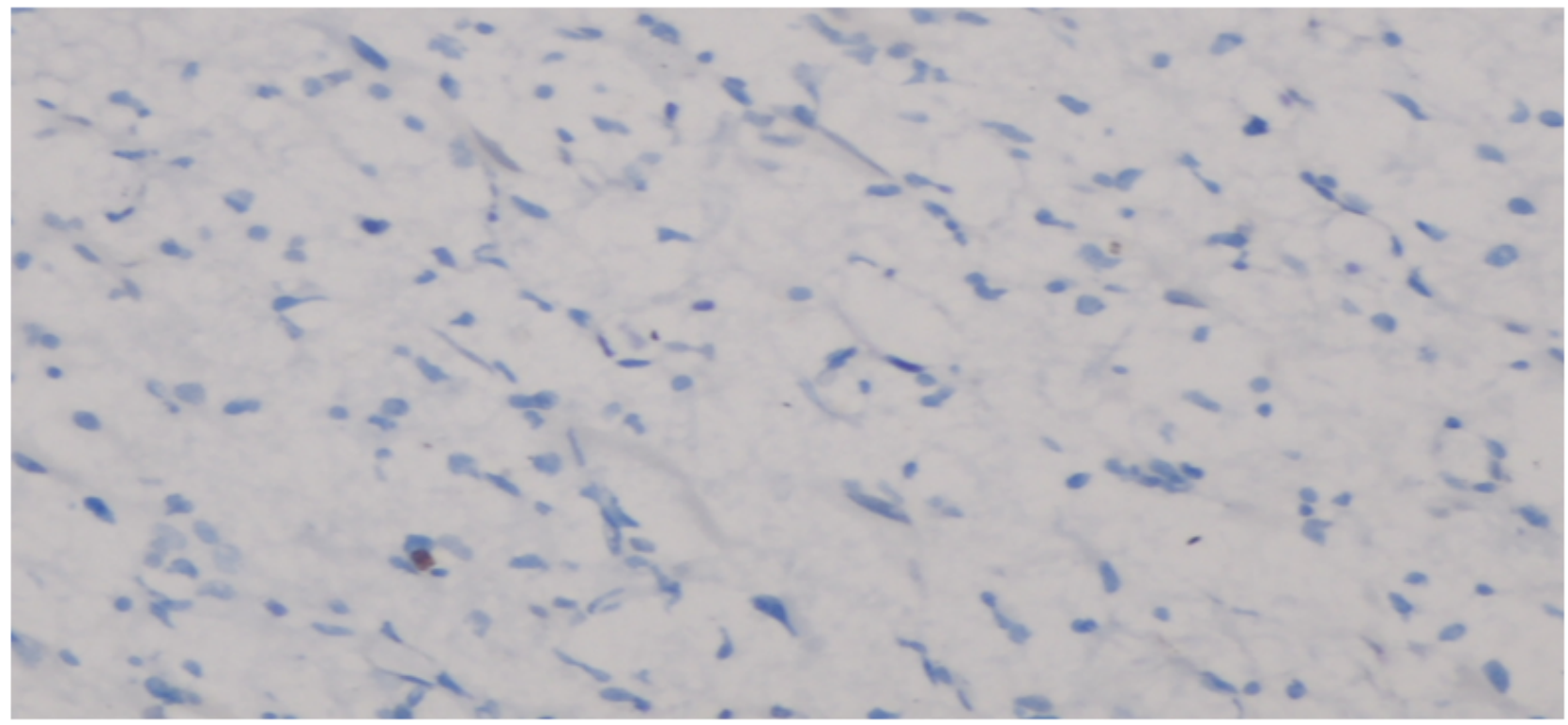

B

Figure 2

(A) by $\mathrm{H}$ and $\mathrm{E}$ in a 41 years old lady in Control group with supraseller meningioma. It shows dense trinuclear neutrophilic infiltration, shedded cytoplasmic vacuoles. Three months follow up was free. (B) of the above mentioned case by immuno-histo-chemistry revealing no PR receptors. 


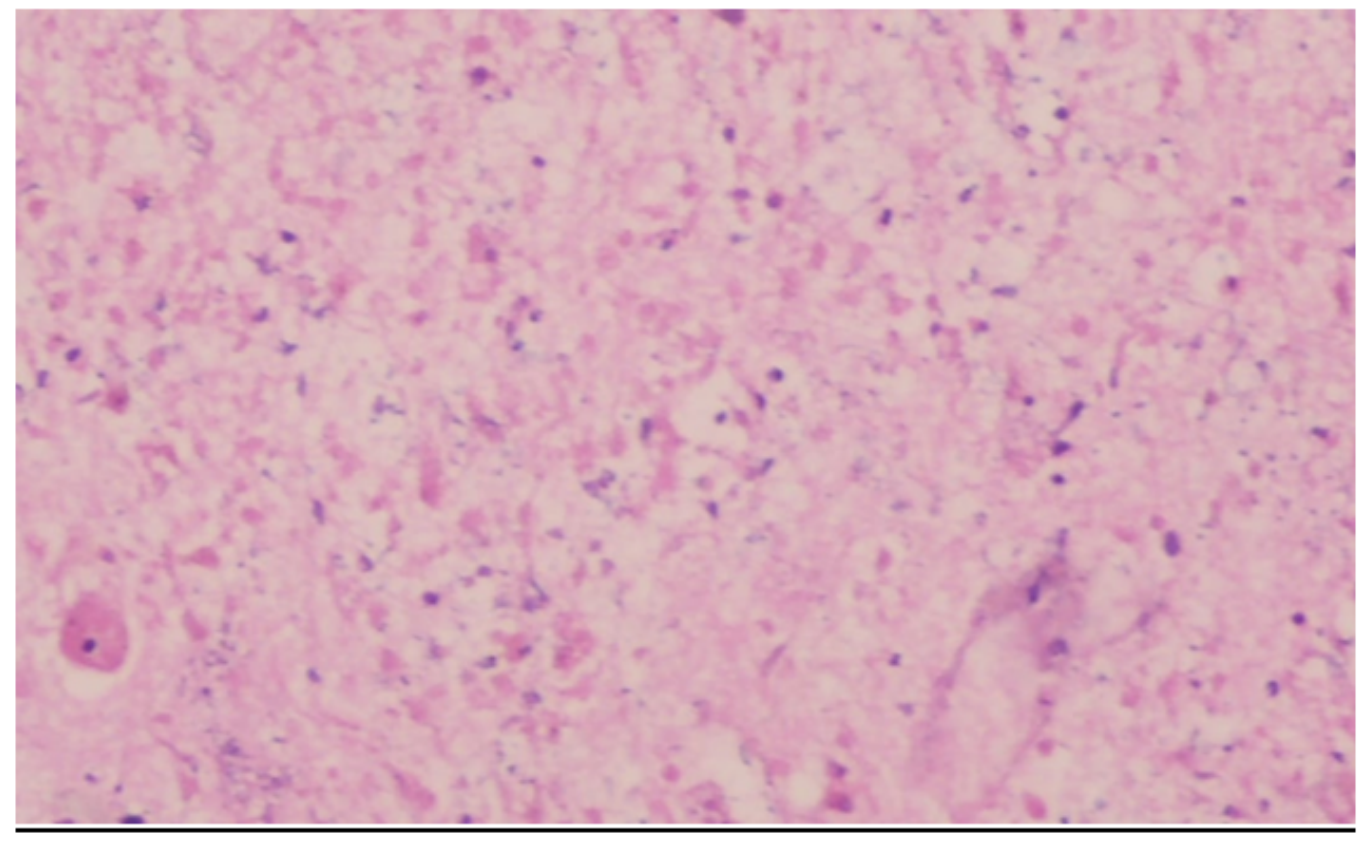

A

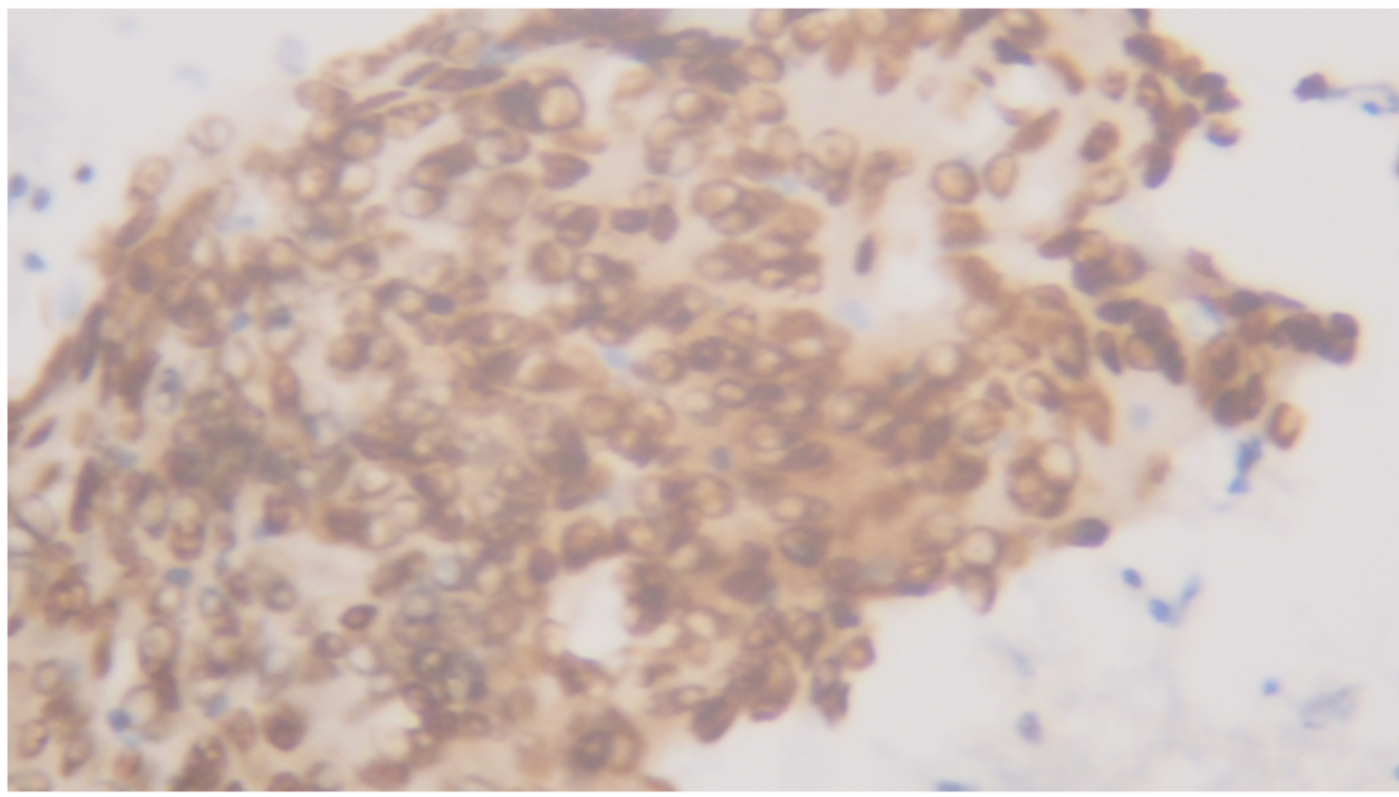

B

\section{Figure 3}

(A) by $\mathrm{H}$ and $\mathrm{E}$ stain of a female patient in PR group with astrocytom (shows completely normal cytoplasm and tightly packed astrocytoma. (B) of the same patient with immuno-histo-chemistry . Dense PR receptors expression. 


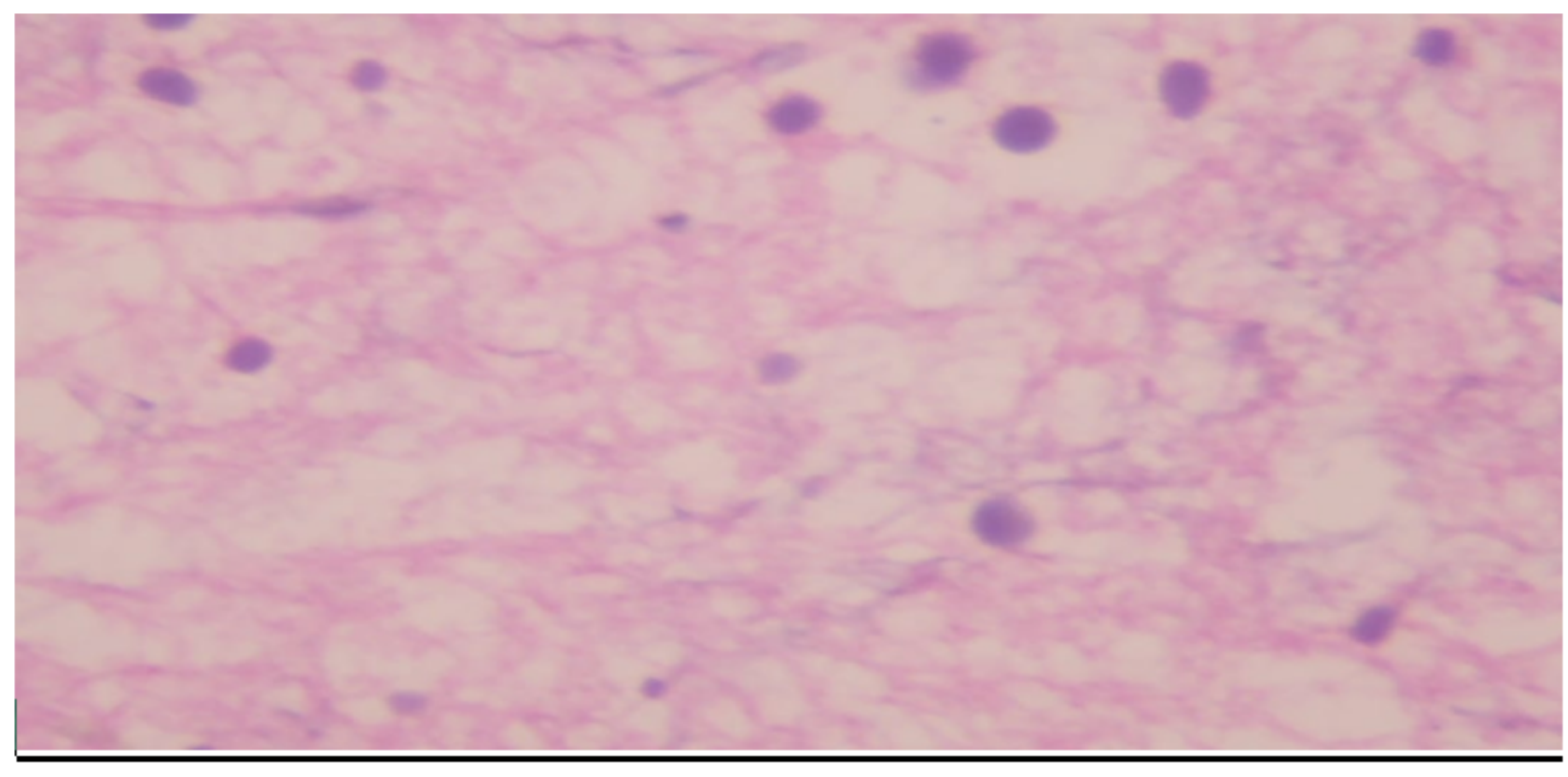

A

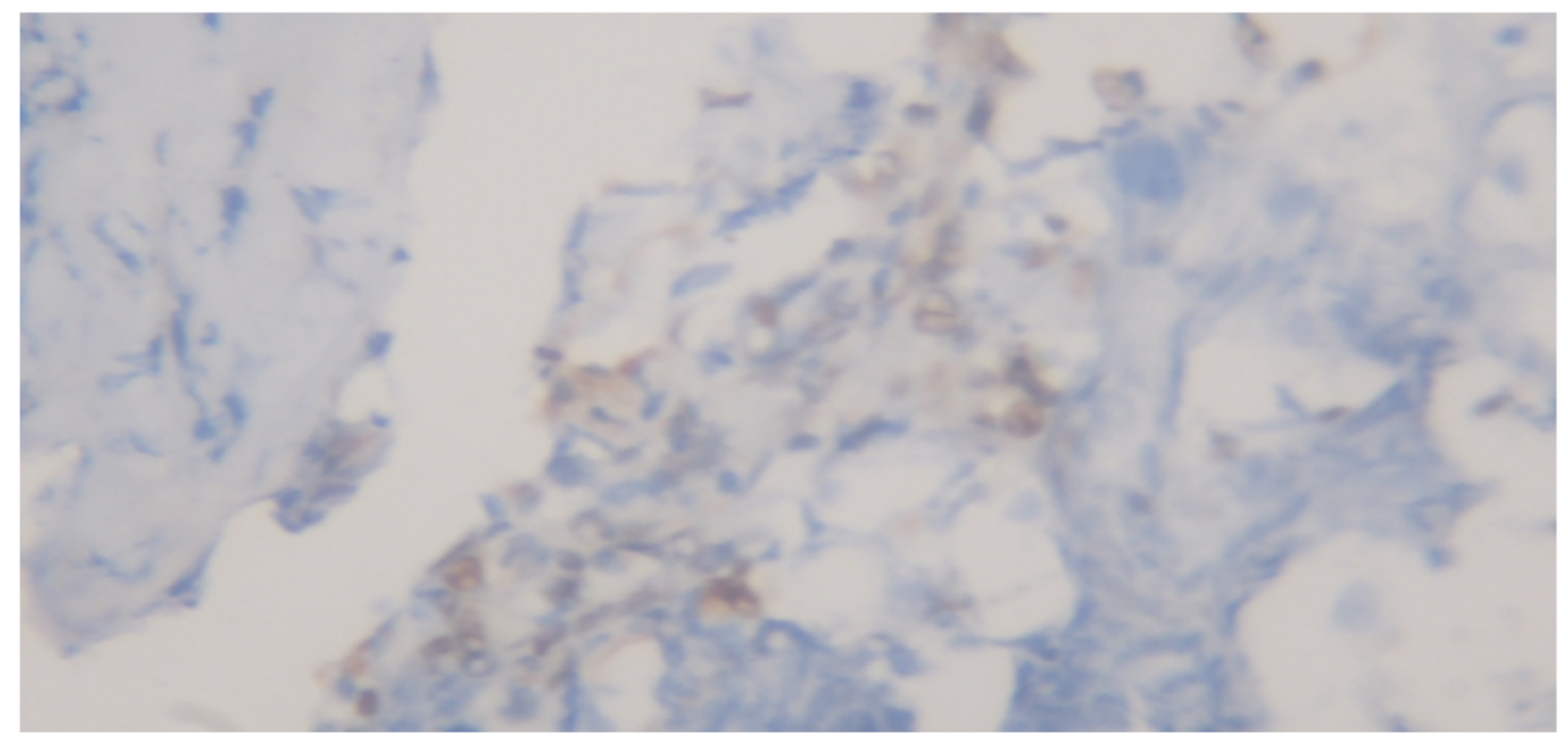

B

\section{Figure 4}

(A) by $\mathrm{H}$ and $\mathrm{E}$ of a male patient in Control group with occipital hemangio-pericytoma shows massive interstial oedema displacing cells from each other. Brain imaging post-craniotomy was free. Three month follow up neurological examination was free. (B) of the same patient by immunohistochemistry showed weak positive PR receptors. 


\section{Supplementary Files}

This is a list of supplementary files associated with this preprint. Click to download.

- Consortflow.doc 